\title{
Evaluation of the microscopic observation drug susceptibility assay for the detection of first- and second-line drug susceptibility for Mycobacterium tuberculosis
}

\author{
To the Editor:
}

The End Tuberculosis Strategy of the World Health Organization (WHO) calls for the early diagnosis of tuberculosis (TB) and use of universal drug susceptibility testing (DST) in order to start patients on the most effective treatment regimen as early as possible. DST using solid or liquid media and rapid molecular-based DST to detect drug-resistant TB are available and endorsed by WHO; however, in 2015, WHO reported that among new cases of bacteriologically confirmed TB, only $12 \%$ had undergone DST [1]. Most national tuberculosis programmes do not offer universal DST [2], and in 2015, 12 countries reported no capacity to perform phenotypic DST [1] while 28 countries had neither in-country capacity nor a linkage with a partner laboratory for second-line DST.

Molecular-based DST is both costly and technically demanding to administer, thus is not widely implemented in many low-income countries [2]. Noncommercial DST methods such as the microscopic observation drug susceptibility (MODS) assay have the potential to help close this gap in diagnostic capacity because they can be implemented in resource-poor settings at low cost and with little training $[3,4]$. MODS has been endorsed by WHO as DST for isoniazid (INH) and rifampicin (RIF) [5], and there is enormous potential for MODS to be used as DST for second-line drugs [6]. The objective of this study was to evaluate the performance of MODS for the simultaneous detection of resistance to seven different anti-TB drugs and to evaluate cut-off points for capreomycin (CAP).

Specimens were collected as part of an observational cohort study (ClinicalTrials registration number NCT02170441) conducted by the Global Consortium for Drug-resistant TB Diagnostics (GCDD). Descriptions of the protocols have been published previously [7].

The Mycobacterium Growth Indicator Tube (MGIT) 960 DST was selected as the phenotypic reference standard, using the critical drug concentrations recommended by the WHO at the time of the study: INH 0.1, RIF 1.0, moxifloxacin (MOX) 0.25, ofloxacin (OFX) 2.0, amikacin (AMK) 1.0, kanamycin (KAN) 2.5 and CAP $2.5 \mu \mathrm{g} \cdot \mathrm{mL}^{-1}[8,9]$.

The MODS assay [6] was performed with drug concentrations of INH 0.4, RIF 1.0, MOX 0.5, OFX 1.0, AMK 2.0 and KAN $5.0 \mu \mathrm{g} \cdot \mathrm{mL}^{-1}[6,10]$, and multiple concentrations of CAP: $1.25,2.5,5.0$ and $10.0 \mu \mathrm{g} \cdot \mathrm{mL}^{-1}$. The pyrosequencing platform $[11,12]$ scanned regions associated with mutations in the rrs (positions 1397-1406) and eis promoter regions (positions -5 to -47 ) to investigate resistance to injectable drugs (KAN, AMK and CAP).

The isolates used by Trollip et al. [6] were not the same samples collected in this prospective study; however, as the methods and staff employed were the same, it was deemed appropriate to pool those results with the current results.

Of 1128 study participants enrolled between April 2012 and June 2013, 213 (18.9\%) were culture-negative, one $(0.1 \%)$ was culture-contaminated and $914(81.0 \%)$ were culture-positive (demographic data have been published previously [7]). In total, 826 (73\%) samples were smear-positive while 302 (27\%) were smear-negative.

@ERSpublications

A new multinational study of the noncommercial MODS assay shows excellent performance in detecting M/XDR-tuberculosis http://ow.ly/FAEJ308OiOw

Cite this article as: Catanzaro DG, Trollip AP, Seifert M, et al. Evaluation of the microscopic observation drug susceptibility assay for the detection of first- and second-line drug susceptibility for Mycobacterium tuberculosis. Eur Respir J 2017; 49: 1602215 [https://doi.org/10.1183/13993003.02215-2016]. 
Of the 1128 MODS tests, 731 (64.8\%) were positive, 288 (25.5\%) were negative, 55 (4.9\%) were contaminated and $43(3.8 \%)$ were indeterminate. $11(1.1 \%)$ had some discordance between the two drug-free control wells. Overall sensitivity for MODS to detect Mycobacterium tuberculosis compared with MGIT was $84.9 \%$ and specificity was $98.8 \%$. For smear-positive samples, overall sensitivity was $91.8 \%$ and specificity was $97.4 \%$, while for smear-negative samples, sensitivity was $46.6 \%$ and specificity was $99.2 \%$.

MODS and MGIT DST results were available for 729 samples. The overall sensitivity was high: INH 97.0\%, RIF 99.6\%, AMK 90.0\%, KAN 61.9\%, MOX 97.8\% and OFX 98.2\%. The overall specificity was excellent: INH 98.7\%, RIF 97.8\%, AMK 99.5\%, KAN 99.8\%, MOX 97.1\% and OFX 98.0\%. Of the 18 non-CAP site/drug combinations, only six had sensitivity lower than 95\% (India-KAN; Moldova-AMK, KAN, MOX, OFX; South Africa-INH) and only four had specificity lower than 98\% (India-INH, MOX, OFX; South Africa-RIF).

All MODS-discordant KAN results in Moldova $(n=40)$ were false positives, of which $26(65 \%)$ had mutations in the eis promoter region scanned. Of the six discordant results in India, two (33\%) had had mutations in the eis promoter region. There were no discordant results for KAN in South Africa, and no South African samples had mutations in the eis promoter region.

Trollip et al. [6] reported provisional cut-off points for CAP, and our results showed that moving from a $2.5 \mu \mathrm{g} \cdot \mathrm{mL}^{-1}$ to a $1.25 \mu \mathrm{g} \cdot \mathrm{mL}^{-1}$ breakpoint yielded an increase of $6 \%$ in sensitivity but a concomitant decrease of $2.1 \%$ in specificity.

Analysis of receiver operating characteristic (ROC) curves (figure 1) showed agreement between this study and two previously published studies on breakpoints using MODS to test for M. tuberculosis resistance to capreomycin [6, 13]. Trollip et al. [6] reported CAP results for four concentrations $(\mathrm{n}=55,29$ of which were CAP-resistant) and FITZWATER et al. [13] reported CAP results for six concentrations ( $\mathrm{n}=94$, of which 51 were CAP-resistant).

ROC curve analysis supported the lowering of the critical concentration CAP for MODS to $1.25 \mu \mathrm{g} \cdot \mathrm{mL}^{-1}$ (figure 1). We pooled our study results with those of Trollip et al. [6], which showed that if the breakpoint concentration for CAP is lowered to $1.25 \mu \mathrm{g} \cdot \mathrm{mL}^{-1}$, a combined sensitivity for CAP of $92.7 \%$ (95\% CI 86-97\%) and specificity of 96.1\% (95\% CI 94-97\%) would be achieved.

This is the first multinational study to prospectively assess the clinical performance of the MODS assay for the detection of multi/extensively resistant TB (M/XDR-TB). The findings from our large study $(\mathrm{n}=1128)$ significantly bolster the current WHO policy guidance, which endorses MODS to screen patients suspected of having MDR-TB [5], and adds to the growing body of literature on the use of MODS to detect resistance to second-line anti-TB drugs.

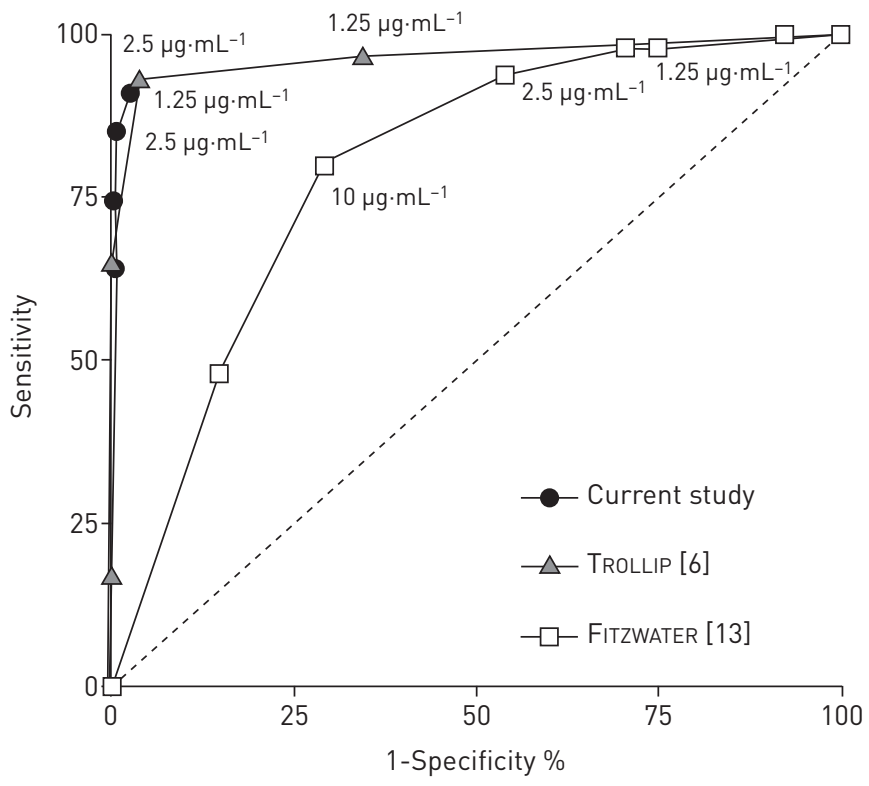

FIGURE 1 Receiver operating characteristic curves for capreomycin. In the current study and that of TROLLIP et al. [6], the reference drug susceptibility testing (DST) method was MGIT960 with a critical concentration of $2.5 \mu \mathrm{g} \cdot \mathrm{mL}^{-1}$ ( $\mathrm{n}=729$ and $\mathrm{n}=55$, respectively); in the study of FitzWATER et al. [13], the reference DST method was a proportional method with an unreported critical concentration $(n=94)$. 
The MODS assay demonstrated a clear dose-response relationship between smear grade/culture and production of a positive, negative or indeterminate result (not shown). The lower sensitivity of MODS could be due to our use of presumptive identification of $M$. tuberculosis with the MODS assay while employing a confirmatory identification of $M$. tuberculosis for our reference standard. It is likely that combining the MODS assay with a test to confirm M. tuberculosis (e.g. para-nitrobenzoic acid or MPT64) would decrease the discordance between MODS and MGIT culture.

In the present study, the $95 \%$ confidence intervals surrounding MODS sensitivity and specificity as a DST for INH, RIF, AMK, MOX and OFX [7] meet and/or exceed the WHO target product profiles for a tuberculosis diagnostic [14]. We found higher sensitivity and specificities than those in the only other prospective study of MODS ( $\mathrm{n}=540$ in one country) for INH, RIF, OFX and CAP, although our estimates were lower for KAN [15]. Our low sensitivity for AMK was driven by a low number of AMK-resistant samples $(n=10)$. Clear performance increases were observed when the CAP breakpoint was lowered to $1.25 \mu \mathrm{g} \cdot \mathrm{mL}^{-1}$.

Investigation of genetic mutations known to confer resistance to KAN revealed that mutation status of samples can have a dramatic effect on accuracy, especially sensitivity, owing to the presence of mutations in $r r s$ and eis promoter regions, which confer high-level ( $r r s$ ) or low-level (eis promoter) resistance to KAN.

The MODS assay yielded comparable results to MGIT first- and second-line DST, and results are obtained much faster than MGIT (but not as fast as the MTBDRsl [7]), thus we believe that MODS can be deployed in resource-poor laboratory settings. Given its noncommercial nature, MODS is a low-cost alternative to other WHO-endorsed DST methods, and operational research would be helpful to determine the cost/benefits of implementing MODS in individual resource-poor laboratory settings.

Donald G. Catanzaro ${ }^{1}$, Andre P. Trollip ${ }^{2}$, Marva Seifert ${ }^{3}$, Sophia B. Georghiou ${ }^{4}$, Richard S. Garfein ${ }^{3}$, Timothy C. Rodwell ${ }^{3}$, Antonino Catanzaro ${ }^{3}$ and Kathleen D. Eisenach ${ }^{5}$

${ }^{1}$ Dept of Biological Sciences, University of Arkansas, Fayetteville, AR, USA. ${ }^{2}$ Foundation for Innovative New Diagnostics, Cape Town, South Africa. ${ }^{3}$ University of California San Diego School of Medicine, La Jolla, CA, USA. ${ }^{4}$ Foundation for Innovative New Diagnostics, Geneva, Switzerland. ${ }^{5}$ University of Arkansas for Medical Sciences, Dept of Pathology, Little Rock, AR, USA.

Correspondence: Donald G. Catanzaro, Dept of Biological Sciences, Science and Engineering, Room 601, University of Arkansas, Fayetteville, AR 72701, USA. E-mail: dgcatanz@uark.edu

Received: Sept 232016 | Accepted after revision: Dec 162016

This study is registered with ClinicalTrials.gov registration number NCT02170441.

Support statement: Data and funding for this project were provided by the NIAID-funded GCDD (grant \#U01-AI082229; PI: A. Catanzaro). T.C. Rodwell was partly funded under NIH grant number P30 AI036214-20 and receives salary support from FIND, a non-profit organisation. The terms of this arrangement have been reviewed and approved by the UCSD. Funding information for this article has been deposited with the Open Funder Registry.

Conflict of interest: Disclosures can be found alongside this article at erj.ersjournals.com

Acknowledgements: The authors would like to acknowledge the site investigators, Valeriu Crudu (Phthisiopneumology Institute), Tommie Victor (Stellenbosch University) and Camilla Rodriques (P.D. Hinduja National Hospital and Medical Research Centre), as well as their clinical and laboratory staff for their assistance in acquiring and testing patient specimens.

\section{References}

1 World Health Organization (WHO). Global Tuberculosis Report 2015. Geneva, World Health Organization, 2015.

2 World Health Organization (WHO). Tuberculosis Diagnostics Technology and Market Landscape. 3rd Edn. UNITAID Secretariat. Geneva, World Health Organization, 2014.

3 Caviedes L, Lee TS, Gilman RH, et al. Rapid, efficient detection and drug susceptibility testing of Mycobacterium tuberculosis in sputum by microscopic observation of broth cultures. The Tuberculosis Working Group in Peru. J Clin Microbiol 2000; 38: 1203-1208.

4 Moore DA, Evans CA, Gilman RH, et al. Microscopic-observation drug-susceptibility assay for the diagnosis of TB. N Engl J Med 2006; 355: 1539-1550.

5 World Health Organization (WHO). WHO policy statement: Noncommercial culture and drug-susceptibility testing methods for screening patients at risk for multidrug-resistant tuberculosis. Geneva, World Health Organization, 2011.

6 Trollip AP, Moore D, Coronel J, et al. Second-line drug susceptibility breakpoints for Mycobacterium tuberculosis using the MODS assay. Int J Tuberc Lung Dis 2014; 18: 227-232.

7 Catanzaro A, Rodwell TC, Catanzaro DG, et al. Performance comparison of three rapid tests for the diagnosis of drug-resistant tuberculosis. PLoS One 2015; 10: e0136861.

8 Rodrigues C, Jani J, Shenai S, et al. Drug susceptibility testing of Mycobacterium tuberculosis against second-line drugs using the Bactec MGIT 960 System. Int J Tuberc Lung Dis 2008; 12: 1449-1455.

9 World Health Organization (WHO). Policy guidance on drug-susceptibility testing (DST) of second-line antituberculosis drugs. Geneva, World Health Organization, 2008. 
10 Park WG, Bishai WR, Chaisson RE, et al. Performance of the microscopic observation drug susceptibility assay in drug susceptibility testing for Mycobacterium tuberculosis. J Clin Microbiol 2002; 40: 4750-4752.

11 Lin SY, Rodwell TC, Victor TC, et al. Pyrosequencing for rapid detection of extensively drug-resistant Mycobacterium tuberculosis in clinical isolates and clinical specimens. J Clin Microbiol 2014; 52: 475-482.

12 Georghiou SB, Seifert M, Catanzaro D, et al. Frequency and distribution of tuberculosis resistance-associated mutations between Mumbai, Moldova, and Eastern Cape. Antimicrob Agents Chemother 2016; 60: 3994-4004.

13 Fitzwater SP, Sechler GA, Jave O, et al. Second-line anti-tuberculosis drug concentrations for susceptibility testing in the MODS assay. Eur Respir J 2013; 41: 1163-1171.

14 World Health Organization (WHO). High-priority target product profiles for new tuberculosis diagnostics: report of a consensus meeting. Geneva, World Health Organization, 2014; p. 98.

15 Huang Z, Qin C, Du J, et al. Evaluation of the microscopic observation drug susceptibility assay for the rapid detection of MDR-TB and XDR-TB in China: a prospective multicentre study. J Antimicrob Chemother 2015; 70: $456-462$. 